\title{
Involvement of Toll-like receptor 4 in vinorelbine-induced vascular endothelial injury
}

\author{
WEIWEI QIAN $^{1 *}$, LIYAN GAO $^{2 *}$, CHONG CHEN $^{1}$, YINGCHUN TAN $^{2}$, YING ZHOU $^{1}$ and ZHENYU LI ${ }^{1}$ \\ ${ }^{1}$ Department of Hematology, The Affiliated Hospital of Xuzhou Medical College; ${ }^{2}$ School of Nursing, \\ Xuzhou Medical College, Xuzhou, Jiangsu 221002, P.R. China
}

Received May 14, 2014; Accepted January 14, 2015

DOI: $10.3892 /$ etm.2015.2494

\begin{abstract}
Vinorelbine (VIN) is a semi-synthetic vinca alkaloid and is one of the most active agents for the treatment of solid tumors. The drug is commonly administered through a peripheral vein. Although VIN is known to cause local venous toxicity, such as drug-induced phlebitis, the mechanism responsible for the toxicity remains unclear. To investigate the role of Toll-like receptor 4 (TLR4) in VIN-induced vascular endothelial injury, human umbilical vein endothelial cells (HUVECs) were prepared from umbilical cords recovered with the written informed consent of the parents and treated with VIN for $60 \mathrm{~min}$. Following the washing away of the VIN, the cells were cultured for a further 6 and $12 \mathrm{~h}$, and the changes in TLR4 expression in the HUVECs were detected by quantitative polymerase chain reaction and western blot analyses. Finally, the effects of VIN on the translocation of nuclear factor $\kappa \mathrm{B}(\mathrm{NF}-\kappa \mathrm{B})$ were determined by immunofluorescence and confocal analysis. The VIN-treated cells were stretched, extended and irregular while the control cells exhibited normal morphology. The TLR4 mRNA and protein levels were significantly higher in the VIN-treated HUVECs than those in the control group $(\mathrm{P}<0.05)$. The rate of $\mathrm{NF}-\kappa \mathrm{B}$ p65 nuclear translocation in the VIN-treated HUVECs was significantly higher than that in the vehicle-treated group $(\mathrm{P}<0.05)$. These results indicated that TLR4 is involved in the development of VIN-induced vascular endothelial injury by affecting the translocation of $\mathrm{NF}-\kappa \mathrm{B}$.
\end{abstract}

Correspondence to: Dr Zhenyu Li or Miss Ying Zhou, Department of Hematology, The Affiliated Hospital of Xuzhou Medical College, 99 West Huaihai Street, Xuzhou, Jiangsu 221002, P.R. China

E-mail: zhenyulimd@163.com

E-mail: zhouying401@yahoo.com.cn

*Contributed equally

Key words: Toll-like receptor 4, vinorelbine, human umbilical vein endothelial cell

\section{Introduction}

Vinorelbine (VIN) is a semi-synthetic vinca alkaloid with an antitumor activity that is associated with its ability to induce the depolymerization of microtubules and disrupt the mitotic spindle apparatus (1). At present, VIN is one of the most active agents used in the treatment of non-small cell lung cancer and other solid tumors (2-4). VIN is typically administered through a peripheral vein (2-4); however, the drug is a moderate vesicant and causes local venous toxicity. The incidence of VIN-induced local venous toxicity has been reported to be $\sim 30 \%(5,6)$. Although infusion phlebitis is common, its pathogenesis is not fully understood; however, it has been suggested that the pathogenesis is associated with chemical irritation of the endothelial cells, leading to sterile inflammation and thrombosis $(7,8)$.

Toll-like receptors (TLRs) are a class of pattern recognition receptors that are important sensors of pathogen invasion. To date, 13 members of the TLR family have been identified in mammals. These TLRs respond to different microbe-associated molecular patterns, including viral and bacterial nucleic acids, lipopolysaccharide (LPS), lipoteichoic acids and flagellin (9), although they can also bind endogenously generated ligands, such as heat shock proteins $(10,11)$, surfactant protein A18 (12), extracellular matrix components (13) and high-mobility group box-1 $(14,15)$. All members of the TLR family, with the exception of TLR3, signal through an adaptor protein known as MyD88, which is responsible for stimulating a cascade of events that leads to the activation of the transcription factor nuclear factor- $\kappa \mathrm{B}(\mathrm{NF}-\kappa \mathrm{B})(16)$.

$\mathrm{NF}-\kappa \mathrm{B}$ is an important regulator of various genes involved in immune and inflammatory responses and is linked to inflammatory and growth responses. TLR4 is known to induce NF- $\kappa \mathrm{B}$ activation; however, it is not yet clear whether VIN induces the activation of the TLR4/NF- $\mathrm{KB}$ pathway in human umbilical vein endothelial cells (HUVECs). Furthermore, the association between the activation of NF- $\mathrm{BB}$ and TLR 4 caused by VIN has yet to be revealed. TLR4 is known to participate in the selective relay of a variety of signals from the membrane to transducers of cell activation and gene expression (16). We postulated that TLR4 was a potential contributing factor in the pathogenesis of VIN-induced vascular endothelial injury. The aim of the present study, therefore, was to examine the effects of VIN on the expression of TLR4 and the transcription factor NF- $\kappa$ B. 


\section{Materials and methods}

HUVEC preparation and culture. HUVECs were prepared using human umbilical veins from umbilical cords recovered with the written informed consent of the parents. Cells were cultured in basal EBM ${ }^{\mathrm{TM}}-2$ basal medium supplemented with growth factor (Lonza Group, Basel, Switzerland) at $37^{\circ} \mathrm{C}$ and under $5 \% \mathrm{CO}_{2}$. EBM-2 basal medium was supplemented with an $\mathrm{EGM}^{\mathrm{TM}}-2 \mathrm{MV}$ SingleQuot ${ }^{\mathrm{TM}}$ kit, also from Lonza Group. Cells from between passages two and five were used.

Exposure ofHUVECs to VIN. Cells at a density of $5 \times 10^{4}$ cells $/ \mathrm{ml}$ were seeded in $25-\mathrm{cm}^{2}$ flasks or $24-w e l l$ plates. Upon reaching 90-95\% confluence, the cells were washed twice with phosphate-buffered saline (PBS) and fed VIN solution at a final concentration of $0.05 \mathrm{mg} / \mathrm{l}$ in the flasks or wells. Untreated control cells were fed medium without VIN. After $1 \mathrm{~h}$ of incubation with VIN, the cells were washed twice with PBS, re-fed medium without VIN and then grown for 0,6 or $12 \mathrm{~h}$. The apoptotic cells were quantified by the Annexin V-PE/7-AAD apoptosis detection kit I (BD Biosciences, Franklin Lakes, NJ, USA). These cells were then washed twice with PBS and harvested for quantitative polymerase chain reaction (qPCR), western blot and immunofluorescence analyses. Experiments were repeated at least three times.

RNA isolation. Cells $\left(10^{6}\right)$ were suspended in $1 \mathrm{ml}$ TRIzol ${ }^{\mathrm{TM}}$ reagent (Invitrogen Life Technologies, Carlsbad, CA, USA) in a 2-ml screw-cap microtube. After $5 \mathrm{~min}$ at room temperature, $0.2 \mathrm{ml}$ chloroform was added and the tube was agitated for $15 \mathrm{sec}$. The tube was then allowed to sit at room temperature for $10 \mathrm{~min}$, prior to centrifugation at $12,000 \mathrm{x} \mathrm{g}$ and $4^{\circ} \mathrm{C}$ for 10 min and the transferral of the supernatant to a Qiagen RNeasy ${ }^{\circledR}$ Mini kit column (Qiagen, Crawley, UK). Total RNA was extracted according to the manufacturer's instructions. RNA samples were stored at $-80^{\circ} \mathrm{C}$ until use.

$q P C R$. Total RNA was extracted from the cells using TRIzol reagent (Invitrogen Life Technologies) following the manufacturer's instructions. The cDNA was generated with an oligo (dt) primer (Invitrogen Life Technologies), according to the manufacturer's instructions. qPCR was performed using the ABI Prism ${ }^{\circledR} 7500$ Sequence Detection System (Applied Biosystems, Branchburg, NJ, USA) with ABI PRISM ${ }^{\circledR}$ 7000 SDS software (Applied Biosystems). PCR was carried out with SYBR ${ }^{\circledR}$ Green PCR Master Mix (Invitrogen Life Technologies) using $1 \mu 1 \mathrm{cDNA}$ in a $25-\mu 1$ final reaction mixture. The reaction mixture was heated initially at $94^{\circ} \mathrm{C}$ for $2 \mathrm{~min}$, followed by 35 cycles of denaturation at $94^{\circ} \mathrm{C}$ for $30 \mathrm{sec}$ and annealing/extension at $60^{\circ} \mathrm{C}$ for $30 \mathrm{sec}$. Data were analyzed using the $2^{-\Delta \Delta \mathrm{CT}}$ method. Glyceraldehyde-3-phosphate dehydrogenase (GAPDH) was utilized for endogenous quantity control. The primers used were as follows: TLR4 forward, 5'-AAGCCGAAAGGTGATTGTTG-3' and reverse, 5'-CTGAGCAGGGTCTTCTCCAC-3'; GAPDH forward, 5'-GGGAAACTGTGGCGTGAT-3' and reverse, 5'-GAG TGGGTGTCGCTGTTGA-3'.

Western blot analysis. Logarithmic growth phase HUVECs were treated with the test compounds or with blank vehicle for the specified lengths of time and then lysed in cell lysis buffer on ice for $40 \mathrm{~min}$. Soluble protein was recovered following centrifugation at $4^{\circ} \mathrm{C}$ at $10,000 \times \mathrm{g}$ for $30 \mathrm{~min}$. Samples were assayed for protein concentration using the bicinchoninic acid assay. The samples were separated on $10 \%$ SDS-PAGE gels and then transferred to nitrocellulose membranes. The membranes were incubated with a polyclonal rabbit anti-human TLR4 antibody (1:500 dilution; cat. no. 2246, Cell Signaling Technology, Inc., Danvers, MA, USA) overnight and then incubated with peroxidase-labeled goat anti-rabbit secondary antibody (1:5,000; cat. no. A0545, Sigma-Aldrich, St. Louis, MO, USA). Mouse polyclonal $\beta$-actin primary antibody was used as a control (1:1,000 dilution; cat. no. 612657, BD Biosciences). Immunoreactive bands were visualized using enhanced chemiluminescence reagents. The protein levels were quantified using Quantity One ${ }^{\circledR}$ software (Bio-Rad, Hercules, CA, USA).

Immunofluorescence and confocal analysis. Cells on coverslips were fixed in $4 \%$ paraformaldehyde for $15 \mathrm{~min}$ at room temperature. Following three washes with PBS, the cells were permeabilized with ice-cold methanol for $10 \mathrm{~min}$ at $-20^{\circ} \mathrm{C}$. The cells were then rinsed three times with PBS and incubated for $1 \mathrm{~h}$ at room temperature in the dark in a PBS solution containing 2\% (wt/v) bovine serum albumin (Merck Millipore, Darmstadt, Germany). Following incubation, the cells were rinsed three more times in PBS and incubated overnight with primary antibodies at $4^{\circ} \mathrm{C}$. The slides were washed a further three times and incubated with Sytox ${ }^{\circledR}$ Green Nucleic Acid Stain (cat. no. S7020; 1:10,000 dilution in Milli-Q water; Invitrogen Life Technologies) at room temperature in the dark for $1 \mathrm{~h}$. Following incubation, the cells were washed three times with PBS. The nuclear dye DAPI was applied and the cells were incubated for $15 \mathrm{~min}$. Following a final wash, the coverslips were mounted in Fluoromount ${ }^{\mathrm{TM}}$ (Vector Laboratories, Inc., Burlingame, CA, USA) and sealed with nail varnish. Stained slides were examined using a Confocal Laser Scanning Biological Microscope (Olympus Fluoview FV1000; Olympus Optical Co., Ltd., Tokyo, Japan) equipped with an Olympus IX70 camera and recorded as a high-resolution layer.

Statistical analysis. Statistical analysis was performed using SPSS 16.0 software (SPSS, Inc., Chicago, IL, USA). Results are expressed the mean \pm standard deviation. Experimental data were analyzed using one-way analysis of variance followed by Tukey's multiple range test for significance. $\mathrm{P}<0.05$ was considered to indicate a statistically significant difference.

\section{Results}

Morphological changes in the HUVECs. HUVECs in the vehicle-treated group showed adherent dermoid growth (Fig. 1), a paving-stone arrangement, spindle-shaped cell morphology and nuclei with abundant cytoplasm. The VIN-treated cells were stretched, extended, irregular and disordered. Once the VIN had been washed away, the cells were cultured for a further 6 and $12 \mathrm{~h}$, during which cell morphology gradually returned to normal. 


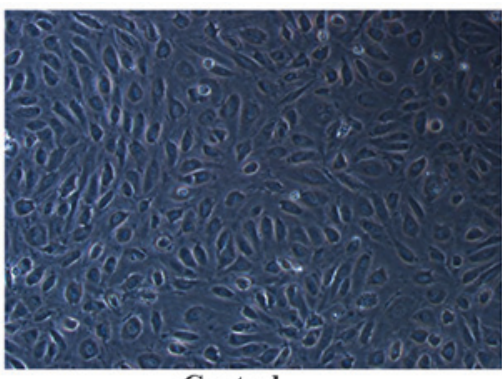

Control

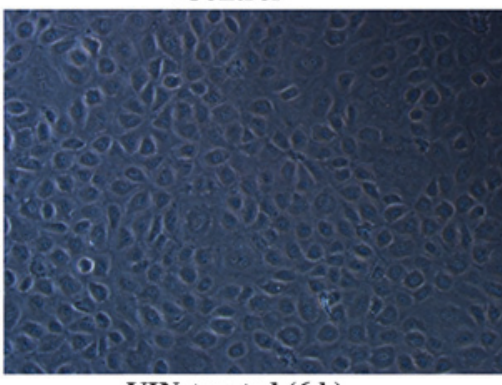

VIN-treated $(6 \mathrm{~h})$

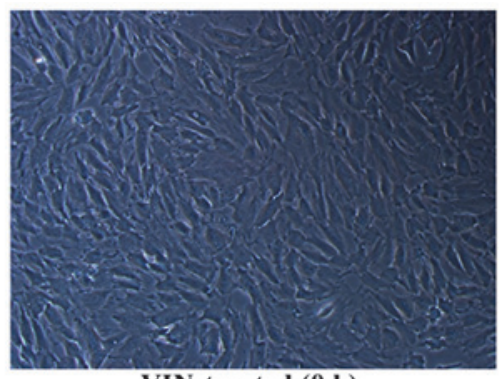

VIN-treated (0 h)

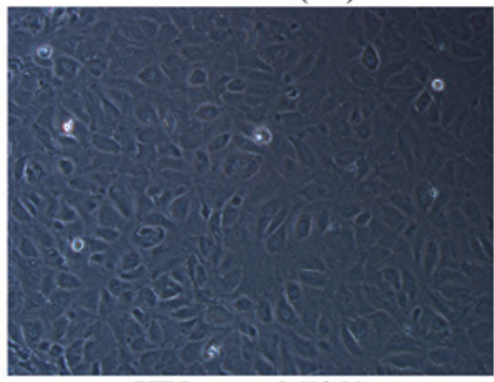

VIN-treated (12 h)

Figure 1. Morphological changes in the HUVECs following VIN treatment. HUVECs in the vehicle-treated group showed adherent dermoid growth, a paving-stone arrangement, spindle-shaped cell morphology and nuclei with abundant cytoplasm (magnification, x100). The VIN-treated cells exhibited stretched, extended, irregular and disordered morphology. The cell damage induced by VIN was reduced as time progressed. VIN, vinorelbine; HUVEC, human umbilical vein endothelial cell.

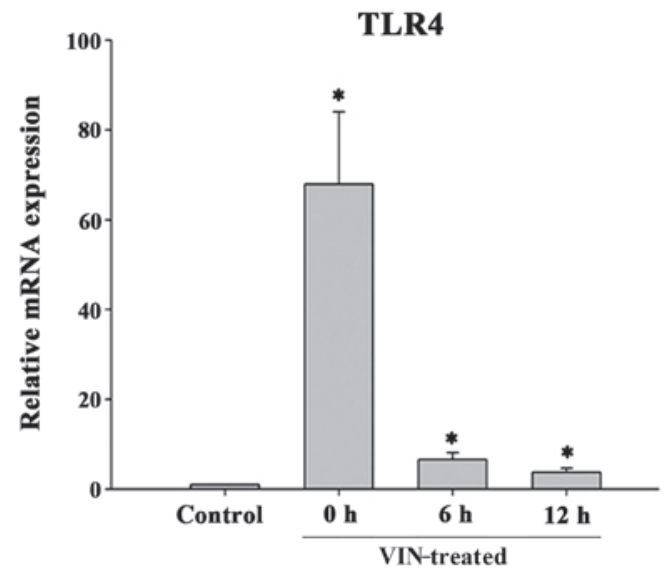

Figure 2. Time course of TLR4 mRNA expression in the HUVECs following VIN treatment. HUVECs were exposed to VIN $(0.05 \mathrm{mg} / \mathrm{l})$ for $1 \mathrm{~h}$ and then washed twice with phosphate-buffered saline, re-fed with medium withou VIN and cultured for 0,6 or $12 \mathrm{~h}$. The control cells were fed medium without VIN only. The mRNA levels of TLR4 were determined using quantitative polymerase chain reaction analysis. Data are expressed as the mean \pm standard deviation $(\mathrm{n}=3)$. ${ }^{*} \mathrm{P}<0.05$ compared with the control group. TLR4 Toll-like receptor 4; HUVEC, HUVEC, human umbilical vein endothelial cell; VIN, vinorelbine.

TLR4 mRNA expression profile in HUVECs exposed to VIN. qPCR was performed to verify the changes in TLR4 mRNA expression in the HUVECs. As shown in Fig. 2, the levels of TLR4 mRNA expression were significantly higher in the VIN-treated HUVECs than those in the vehicle-treated group $(\mathrm{P}<0.05)$. The effects of VIN peaked $1 \mathrm{~h}$ after initial exposure.

TLR4 protein expression profile of HUVECs exposed to VIN. Western blotting was performed to assess the relative levels of TLR4 protein expression in the HUVECs and determine the

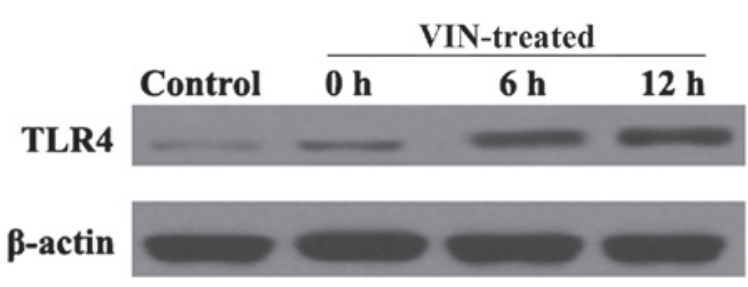

Figure 3. Western blot analysis of TLR4 expression in HUVECs following VIN treatment. HUVECs were exposed to VIN $(0.05 \mathrm{mg} / \mathrm{l})$ for $1 \mathrm{~h}$, and then washed twice with phosphate-buffered saline, re-fed with medium without VIN and cultured for 0,6 or $12 \mathrm{~h}$. The control cells were fed medium without VIN only. TLR4, Toll-like receptor 4; HUVEC, HUVEC, human umbilical vein endothelial cell; VIN, vinorelbine.

effects of VIN treatment. As shown in Fig. 3, the level of TLR4 protein expression in the VIN-treated groups was significantly higher than that in the vehicle-treated group.

Effects of VIN on the activation of $N F-\kappa B$ p65. Given the crucial role attributed to VIN in the induction of vascular endothelial damage, the next focus of the study was on $\mathrm{NF}-\kappa \mathrm{B}$ signaling. As shown in Fig. 4, NF- $\kappa \mathrm{B}$ activation, as indicated by $\mathrm{NF}-\kappa \mathrm{B}$ phosphorylation, was assessed at different times and became undetectable $20 \mathrm{~h}$ after treatment (data not shown). As expected, nuclear translocation of p65 occurred in the HUVECs treated with VIN, and NF- $\mathrm{B}$ activation occurred within a narrow window in the HUVECs subjected to vascular endothelial injury.

Effects of VIN on apoptosis. VIN-induced apoptosis was quantified using Annexin V/7-AAD. The results indicated that the apoptotic rate of the HUVECs was increased in response to VIN, in a dose-dependent manner (data not shown). This result suggests that the increased apoptosis of the HUVECs 


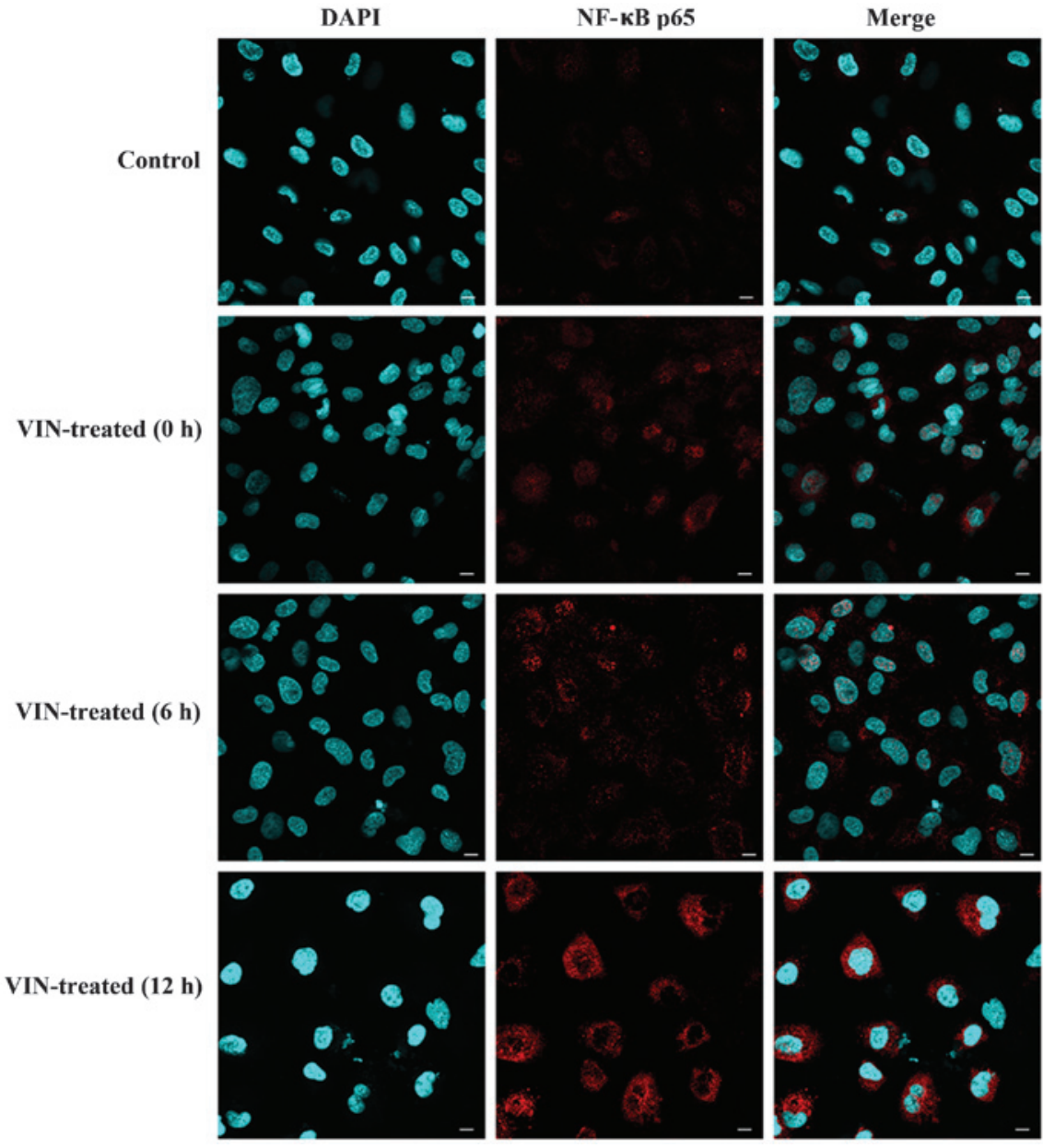

Figure 4. Immunofluorescence and confocal analysis of NF- $\mathrm{B}$ p65 expression in HUVECs following the VIN treatment. Staining was performed with an

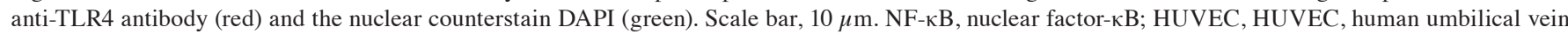
endothelial cell; VIN, vinorelbine; TLR4, Toll-like receptor 4.

had a close association with the decreased expression levels of TLR4, as caused by treatment with VIN.

\section{Discussion}

Vascular endothelial cells adhere to the vascular walls, forming an integral part of the blood vessels and exhibiting a variety of immunological functions. The dysfunction of endothelial cell apoptosis has been observed in human diseases and experimental models, including atherosclerosis, ischemia-reperfusion and sepsis (17-19). VIN is a moderate vesicant that has been known to cause local venous toxicity $(3,6)$. Although the pathogenesis of VIN has been little reported, it has previously been suggested that improper endothelial cell apoptosis could be responsible for the local venous toxicity caused by the VIN (19). Thus, primary HUVECs were selected for the present study in order to reveal their roles in the protection against vascular endothelial injury.

The morphology experiments suggested that VIN significantly changed the morphology of the HUVECs, causing them to stretch, extend and become irregular and disordered. The results indicated that the VIN-induced HUVEC injury involved disruption of the morphological structure of the cell and that the removal of the stimuli allowed the cells to self-regulate and return to their normal state. Furthermore, consistent with the present findings, it has previously been shown that VIN can induce apoptotic cell death in HUVECs (19). Thus, the present and previous findings have indicated that endothelial cell injury can contribute to VIN-induced vascular injury.

In a previous study by Frantz et al (20) it was found that TLR4 was constitutively expressed in cardiac myocytes and that an upregulation of TLR4 expression was present in the hearts of humans with cardiomyopathies and rodents with experimental cardiac dysfunction. Furthermore, in cultured vascular endothelial cells TLR4 expression has been shown to increase from baseline to high levels following stimulation with proinflammatory cytokines $(21,22)$.

Several studies have suggested that, when tissue is damaged, a variety of endogenous ligands are produced, which can stimulate and promote the expression of TLR4 $(3,13)$. Vascular endothelial cell apoptosis and injury are involved in the formation of phlebitis $(19,21)$. This may indicate that the endogenous ligands are released following endothelial cell injury, possibly leading to the upregulation of TLR4 in the endothelial cells $(19,21)$. The qPCR and western blot analysis showed that TLR4 mRNA and protein levels were significantly higher in the VIN-treated cells than those in the control group. These results indicate that TLR4 is involved in VIN-induced vascular endothelial injury by affecting gene transcription and 
protein synthesis. This result is consistent with the finding of previous studies that VIN stimulation can lead to the upregulation of TLR4 in endothelial cells $(19,20,22)$.

$\mathrm{NF}-\kappa \mathrm{B}$ plays a central role in inflammation through its ability to induce the transcription of proinflammatory genes (23). In resting cells, $\mathrm{NF}-\kappa \mathrm{B}$ is localized in the cytoplasm due to the binding of inhibitor of $\kappa \mathrm{B}(\mathrm{I} \kappa \mathrm{B})$ proteins (23). The induction of $\mathrm{NF}-\kappa \mathrm{B}$ activation by TLR4 is dependent upon the phosphorylation of I $\kappa \mathrm{B} \alpha$ mediated by the I $\mathrm{B}$ kinases IKK $\alpha$ and IKK $\beta$. I $\kappa \mathrm{B} \alpha$ phosphorylation stimulates its ubiquitination and proteasomal degradation, thus releasing $\mathrm{NF}-\kappa \mathrm{B}$ and enabling its translocation to the nucleus, where it can activate the transcription of genes encoding inflammatory molecules (24).

A previous study suggested that $N F-\kappa B$ may contribute to endothelial cell injury by affecting inflammatory molecules (23). As $N F-\kappa B$ p65 is representative of the $N F-\kappa B$ family (14), the effect of VIN on the activation of NF- $\kappa B$ p65 was investigated in the present study. The results showed that VIN promoted NF- $\kappa \mathrm{B}$ p 65 activation and translocation to the nucleus, which stimulated the release of a range of inflammatory factors. The activation of the $\mathrm{NF}-\kappa \mathrm{B}$ pathway is adapted to the upregulation of numerous immune and stress response genes, which terminate the activation (24). The rates of translocation of NF-кB p65 and the expression level of TLR4 mRNA initially increased in the VIN-treated cells, then decreased.

In a study by Frantz et al (20) it was indicated that a 'basal' level of NF- $\mathrm{NB}$ activity was necessary for the maintenance of TLR4 expression. Specifically, it was suggested that ammonium pyrrolidine dithiocarbamate could reproducibly suppress TLR4 mRNA and protein abundance in the cells of both normal and failing myocardium, even in the absence of interleukin (IL)-1 and LPS (20). For this reason, we hypothesized that the activity of NF- $\kappa \mathrm{B}$ plays an important role in the regulation of TLR4. TLR4 activation can also activate $\mathrm{NF}-\kappa \mathrm{B}$, suggesting that there may be a positive feedback mechanism between the two. The fact that TLR4 activates the NF- $\mathrm{B}$ pathway has been known for some time, but the manner in which NF- $\mathrm{BB}$ regulates TLR4 expression is not clear, and further investigation is required.

In conclusion, the present study showed that exposure of HUVECs to $0.05 \mathrm{mg} / \mathrm{l}$ VIN upregulates TLR4 mRNA and protein expression, activating $\mathrm{NF}-\kappa \mathrm{B}$ p65. These results suggest that VIN induces HUVEC injury, possibly via the TLR4/NF- $\mathrm{B}$ pathway. TLR4 is stimulated by VIN and signals to the transcription factor $N F-\kappa B$, which regulates the expression of numerous inflammatory cytokines and chemokines. Our data may provide a novel mechanism for VIN-induced phlebitis. VIN can injure HUVECs, releasing a variety of signals associated with danger and death. This in turn can activate TLR4 and elevate the activity of the NF- $\kappa \mathrm{B}$ pathway. To the best of our knowledge, this is the first report to describe a role for TLR4 in VIN-induced phlebitis in vitro. In this way, this study provides novel experimental evidence regarding potential therapeutic targets for the prevention and treatment of VIN-induced phlebitis. Further study is required to determine the specific role of TLR4 in VIN-induced vascular endothelial injury.

\section{Acknowledgements}

The authors would like to thank Professor Hongyan Dong and Dr Qingyun Wu (Department of Hematology, The Affiliated
Hospital of Xuzhou Medical College, Xuzhou, China) for their technical assistance.

\section{References}

1. Potier P: The synthesis of Navelbine prototype of a new series of vinblastine derivatives. Semin Oncol 16 (2 Suppl 4): 2-4, 1989.

2. Depierre A, Lemarie E, Dabouis G, et al: A phase II study of Navelbine (vinorelbine) in the treatment of non-small-cell lung cancer. Am J Clin Oncol 14: 115-119, 1991.

3. Fumoleau P, Delgado FM, Delozier T, et al: Phase II trial of weekly intravenous vinorelbine in first-line advanced breast cancer chemotherapy. J Clin Oncol 11: 1245-1252, 1993.

4. Devizzi L, Santoro A, Bonfante V, et al: Vinorelbine: a new promising drug in Hodgkin's disease. Leuk Lymphoma 22: 409-414, 1996.

5. Yoh K, Niho S, Goto K, et al: High body mass index correlates with increased risk of venous irritation by vinorelbine infusion. J Clin Oncol 34: 206-209, 2004.

6. Yoh K, Niho S, Goto K, et al: Randomized trial of drip infusion versus bolus injection of vinorelbine for the control of local venous toxicity. Lung Cancer 55: 337-341, 2007.

7. Lewis GB and Hecker JF: Infusion thrombophlebitis. Br J Anaesth, 57: 220-233, 1985.

8. Falchuk KH,Peterson L and McNeil BJ: Microparticulate-induced phlebitis. Its prevention by in-line filtration. N Engl J Med 312: 78-82, 1985.

9. Miyake K: Innate immune sensing of pathogens and danger signals by cell surface Toll-like receptors. Semin Immunol 19: 3-10, 2007.

10. Ohashi K, Burkart V, Flohé S and Kolb H: Cutting edge: heat shock protein 60 is a putative endogenous ligand of the toll-like receptor-4 complex. J Immunol 164: 558-561, 2000.

11. Vabulas RM, Braedel S, Hilf N, et al: The endoplasmic reticulum-resident heat shock protein Gp96 activates dendritic cells via the Toll-like receptor 2/4 pathway. J Biol Chem 277: 20847-20853, 2002.

12. Roelofs MF, Boelens WC, Joosten LA, et al: Identification of small heat shock protein B8 (HSP22) as a novel TLR4 ligand and potential involvement in the pathogenesis of rheumatoid arthritis. J Immunol 176: 7021-7027, 2006.

13. Guillot L, Balloy V, McCormack FX, et al: Cutting edge: the immunostimulatory activity of the lung surfactant protein-A involves Toll-like receptor 4. J Immunol 168: 5989-5992, 2002.

14. Rakoff-Nahoum S and Medzhitov R: Regulation of spontaneous intestinal tumorigenesis through the adaptor protein MyD88. Science 317: 124-127, 2007.

15. Park JS, Svetkauskaite D, He Q, et al: Involvement of toll-like receptors 2 and 4 in cellular activation by high mobility group box 1 protein. J Biol Chem 279: 7370-7377, 2004.

16. Kawai T and Akira S: TLR signaling. Semin Immunol 19: 24-32, 2007.

17. Tricot O, Mallat Z, Heymes C, et al: Relation between endothelial cell apoptosis and blood flow direction in human atherosclerotic plaques. Circulation 101: 2450-2453, 2000.

18. Scarabelli TM, Stephanou A, Pasini E, et al: Different signaling pathways induce apoptosis in endothelial cells and cardiac myocytes during ischemia/reperfusion injury. Circ Res 90: 745-748, 2002.

19. Bannerman DD and Goldblum SE: Mechanisms of bacterial lipopolysaccharide induced endothelial apoptosis. Am J Physiol Lung Cell Mol Physiol 284: L899-L914, 2003.

20. Frantz S, Kobzik L, Kim YD, et al: Toll4 (TLR4) expression in cardiac myocytes in normal and failing myocardium. J Clin Invest 104: 271-280, 1999.

21. Faure E, Equils O, Sieling PA, et al: Bacterial lipopolysaccharide activates NF-kappaB through toll-like receptor 4 (TLR-4) in cultured human dermal endothelial cells. Differential expression of TLR-4 and TLR-2 in endothelial cells. J Biol Chem 275: 11058-11063, 2000.

22. Faure E, Thomas L, Xu H, et al: Bacterial lipopolysaccharide and IFN-gamma induce Toll-like receptor 2 and Toll-like receptor 4 expression in human endothelial cells: role of NF-kappa B activation. J Immunol 166: 2018-2024, 2001.

23. Barnes PJ and Karin M: Nuclear factor-kappaB: A pivotal transcription factor in chronic inflammatory diseases. N Engl J Med 336: 1066-1071, 1997.

24. Winn RK and Harlan JM: The role of endothelial cell apoptosis in inflammatory and immune diseases. J Thromb Haemost 3: $1815-1824,2005$ 